\title{
Posterior interhemispheric transfalcine transprecuneus approach for microsurgical resection of periatrial lesions: indications, technique, and outcomes
}

\author{
Bradley N. Bohnstedt, MD, Charles G. Kulwin, MD, Mitesh V. Shah, MD, and \\ Aaron A. Cohen-Gadol, MD, MSc \\ Goodman Campbell Brain and Spine, Indiana University Department of Neurological Surgery, Indianapolis, Indiana
}

\begin{abstract}
OBJECT Surgical exposure of the peritrigonal or periatrial region has been challenging due to the depth of the region and overlying important functional cortices and white matter tracts. The authors demonstrate the operative feasibility of a contralateral posterior interhemispheric transfalcine transprecuneus approach (PITTA) to this region and present a series of patients treated via this operative route.
\end{abstract}

METHODS Fourteen consecutive patients underwent the PITTA and were included in this study. Pre- and postoperative clinical and radiological data points were retrospectively collected. Complications and extent of resection were reviewed.

RESULTS The mean age of patients at the time of surgery was 39 years (range 11-64 years). Six of the 14 patients were female. The mean duration of follow-up was 4.6 months (range 0.5-19.6 months). Pathology included 6 arteriovenous malformations, 4 gliomas, 2 meningiomas, 1 metastatic lesion, and 1 gray matter heterotopia. Based on the results shown on postoperative MRI, 1 lesion (7\%) was intentionally subtotally resected, but $\geq 95 \%$ resection was achieved in all others (93\%) and gross-total resection was accomplished in $7(54 \%)$ of 13 . One patient (7\%) experienced a temporary approach-related complication. At last follow-up, 1 patient (7\%) had died due to complications of his underlying malignancy unrelated to his cranial surgery, 2 (14\%) demonstrated a Glasgow Outcome Scale (GOS) score of 4, and 11 (79\%) manifested a GOS score of 5 .

CONCLUSIONS Based on this patient series, the contralateral PITTA potentially offers numerous advantages, including a wider, safer operative corridor, minimal need for ipsilateral brain manipulation, and better intraoperative navigation and working angles.

http://thejns.org/doi/abs/10.3171/2015.3.JNS14847

KEY WORDS arteriovenous malformation; tumor; surgical approach; atrium; trigone; interhemispheric approach; microsurgical resection; surgical technique

$\mathrm{P}$ ERITRIGONAL lesions pose special neurosurgical challenges for their exposure and resection because of their proximity to critical structures, including the overlying cortices and white matter fiber tracts. The anterior and posterior choroidal, pericallosal, and splenial arteries provide blood flow to the surrounding normal parenchyma as well as to lesions in this area. There is also significant deep venous drainage via the indispensable internal cerebral veins, vein of Rosenthal, and the straight sinus. Highly functional cerebral cortex and white matter tracts in this area include the optic radiations lateral to the ventricle,${ }^{20}$ postcentral gyrus supralaterally, and the thala- mus anteroinferiorly. ${ }^{13}$ These structures significantly limit the surgical corridor to the peritrigonal area. With the use of standard interhemispheric or transcortical approaches, there is a need for significant retraction/disruption and resection of normal parenchyma and white matter tracts to reach such lesions; these approaches carry a risk of morbidity to the patient.

A wide variety of approaches have been described to facilitate relatively safe lesion exposure while dealing with the surrounding normal anatomy. In 1854, Shaw ${ }^{26}$ described the first approach to this area for a meningioma. Subsequent approaches required an anterior-inferior

ABBREVIATIONS AVM = arteriovenous malformation; GOS = Glasgow Outcome Scale; PITTA = posterior interhemispheric transfalcine transprecuneus approach. ACCOMPANYING EDITORIAL See pp 1042-1044. DOI: 10.3171/2014.10.JNS142002.

SUBMITTED April 14, 2014. ACCEPTED March 19, 2015.

INCLUDE WHEN CITING Published online May 1, 2015; DOI: 10.3171/2015.3.JNS14847.

DISCLOSURE Dr. Shah has served as a consultant to Stryker. 
temporal resection, a posterior-inferior temporal resection, a parahippocampal resection, a paramedian posterior parietal resection, a parasagittal resection, and cingulate resection. ${ }^{9}$ Wang and colleagues, ${ }^{30}$ through their cadaveric study, described a contralateral posterior interhemispheric transfalcine transprecuneus approach (PITTA) to the atrium that offers numerous hypothetical advantages, including a wider operative corridor, minimal ipsilateral brain manipulation, and better intraoperative navigation due to decreased perilesional parenchymal retraction. He$\operatorname{ros}^{10}$ rightfully questioned the application of this operative approach because of its associated risks, but considered it potentially feasible.

Although $\mathrm{Goel}^{8}$ initially described the contralateral transfalcine route for tumor resection, he used this method for a medial frontal lesion accompanied by significant ipsilateral edema, interfering with the tumor's ipsilateral exposure. A transfalcine approach to avoid ipsilateral retraction on a vascular lesion has also been described for anterior interhemispheric pathology. ${ }^{6}$

More recently, Zaidi et al. ${ }^{33}$ described contralateral transcallosal and transcingulate approaches through the lateral ventricle for resection of deep-seated (thalamic and basal ganglion) cavernous malformations. To our knowledge, there are only 2 case reports ${ }^{34}$ but no patient series in the literature describing the operative details of the contralateral posterior interhemispheric transprecuneus route for periatrial or trigonal lesions. We present 14 consecutive cases managed microsurgically via the contralateral PITTA. We will outline the associated challenges and advantages of this operative route. The clinical details of 2 representative patients will also be presented.

\section{Methods}

We performed a retrospective review of the medical records of all pertinent surgeries performed by the senior author (A.A.C.G.) at Methodist Hospital, Indianapolis, Indiana, from November 2010 through February 2014. Fourteen patients underwent resection of their periatrial lesions through the contralateral PITTA. Furthermore, all patients underwent the same exact operative exposure for access to their lesions. Pre- and postoperative clinical and radiological data were collected with particular attention to approach-related and resection-related complications and extent of resection. Each patient's Glasgow Outcome Scale (GOS) score at the last follow-up evaluation was also recorded. The Indiana University Institutional Review Board approved this retrospective review of the patient data.

During this 4-year interval, the senior author used this approach for all periatrial lesions, except for 1 large metastatic tumor that had extended more anteriorly and had displaced the posterior thalamus. This lesion was deemed unsuitable for this approach due to its significant anterior progression into the diencephalon. We employed the posterior contralateral interhemispheric route for lesions confined to the medial wall of the atrium as well as within the trigone of the ventricle. Significant anterior or lateral extension of the lesion into the posterior diencephalon or lateral walls of the atrium, respectively, were considered contraindications for use of the approach.

\section{Preoperative and Postoperative Clinical and Radiological Evaluation}

All patients underwent preoperative and postoperative imaging. If visual dysfunction was among patients' presenting symptoms or detected on a detailed confrontational visual field examination, they also underwent an ophthalmological evaluation. All patients, except 1 who underwent subtotal resection of his periventricular heterotopia (Case 13, Table 1), were evaluated by postoperative MRI or cerebral arteriograms within 24 hours after surgery to assess the extent of resection of their tumors or arteriovenous malformations (AVMs), respectively. Any postoperative new neurological deficit or asymptomatic imaging findings related to the contralateral, unaffected, or "approach" hemisphere were recorded as an "approachrelated" complication. Any other complication was recorded as "resection-related."

\section{Operative Technique}

The patient is placed in a three-quarter prone position with the ipsilateral hemisphere harboring the lesion in the nondependent (up) position and the normal, unaffected or "approach" hemisphere in the dependent (down) position to facilitate the use of gravity retraction to mobilize the normal contralateral hemisphere away from the falx, thereby avoiding the need for fixed rigid retractors on the normal hemisphere (Fig. 1A-C). The patient's head is rotated so that the axis of the superior sagittal sinus makes a $45^{\circ}$ angle with the floor. Neuronavigation guidance is used in every case to assist with mapping the craniotomy flap, avoiding the parasagittal bridging veins, using a precise trajectory of approach, and making an accurate corticotomy site at the precuneus. A lumbar drain is placed to drain CSF and minimize retraction on the unaffected contralateral hemisphere. We did not use neurophysiological monitoring.

We use a small linear incision perpendicular to the superior sagittal sinus over the superior parietal lobule (Fig. 1C). The craniotomy (often $4 \times 3 \mathrm{~cm}$ ) is completed on the contralateral parietooccipital region while the important bridging veins in the region are monitored and therefore avoided as guided by neuronavigation (Fig. 1D). A posteriorly located craniotomy over the occipital lobe is avoided to protect the cortices responsible for vision. Similarly, a more posterior frontal or anterior parietal craniotomy is avoided to prevent exposure and injury to the sensorimotor cortex. After placement of 2 bur holes over the superior sagittal sinus, a parasagittal craniotomy is elevated with exposure of the corresponding length and entire width of the dural sinus. The dura is opened in a horseshoe-shaped fashion based on the shape of the sinus (Fig. 1E and F). Two retraction or tack-up sutures are placed through the superior portion of the falx just below the sinus, allowing for gentle retraction of the falx and rotation of the dural venous sinus, therefore extending the operative corridor medially (Fig. $1 \mathrm{G}$ and $\mathrm{H}$ ). Parasagittal bridging veins may need to be untethered microsurgically to expand the interhemispheric operative view anteriorly or posteriorly.

Approximately $50-60 \mathrm{ml}$ of CSF is gradually removed (in 10-15-ml aliquots) through the lumbar drain after dural opening to maximize cerebral relaxation before in- 


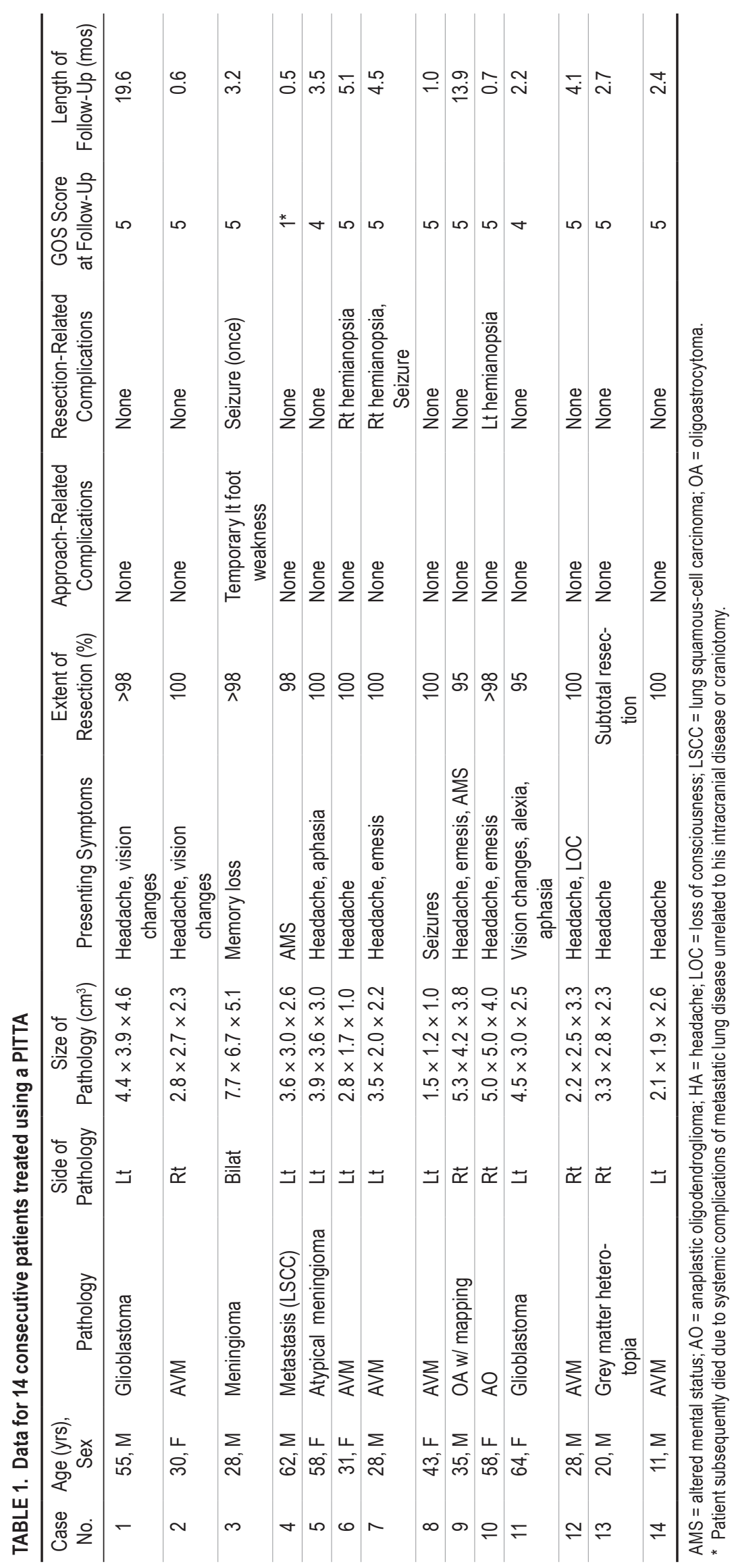



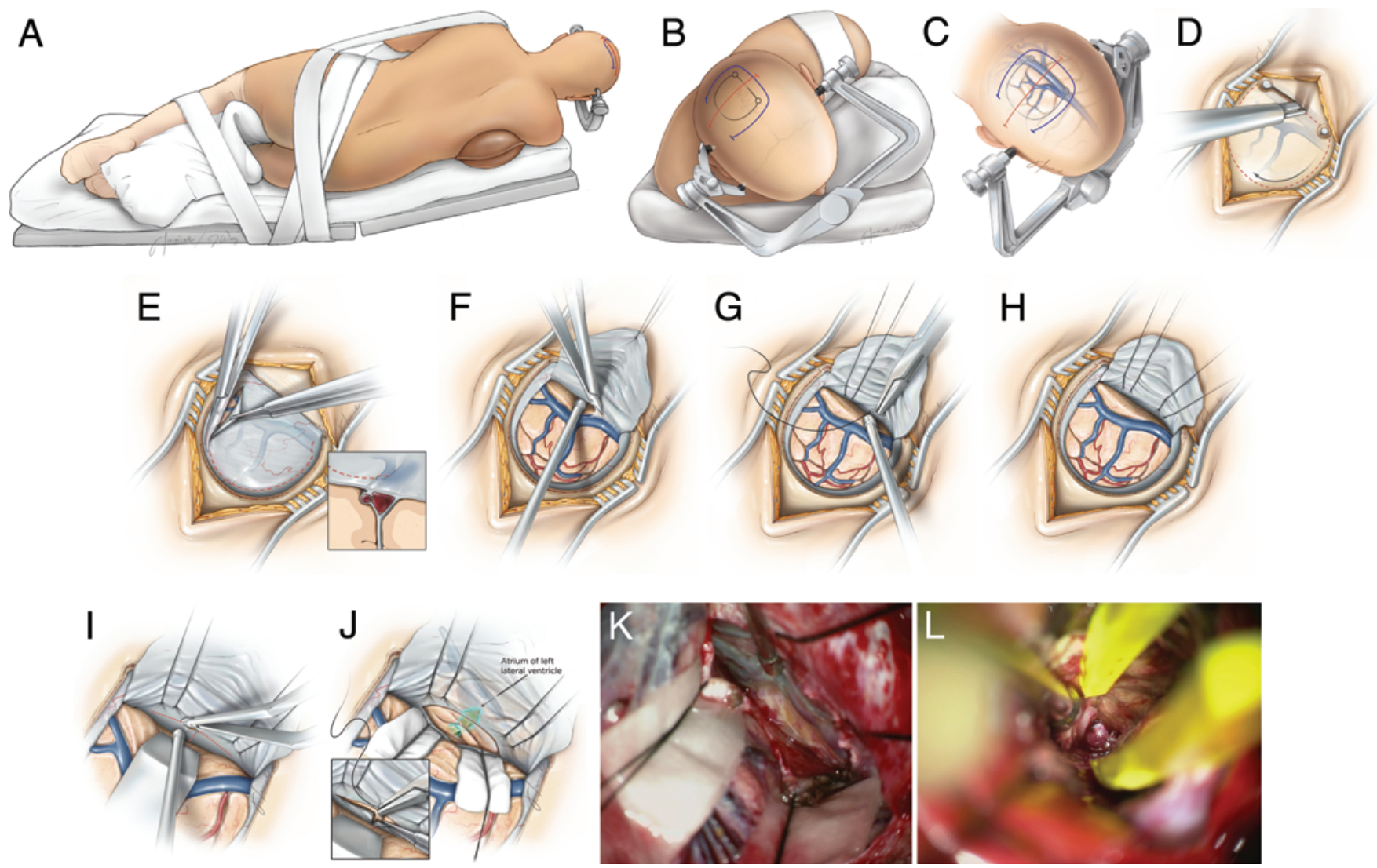

FIG. 1. Illustrations and intraoperative photographs of the procedure. A-C: Patient position and various incision options for approaching a left periatrial lesion while placing the contralateral "approach" hemisphere in the more dependent position to use gravity retraction. D: Bur holes are placed on the superior sagittal sinus and a parasagittal craniotomy is completed while exposing the corresponding segment of the dural sinus. $\mathrm{E}$ : A dural opening is made while avoiding the important bridging veins in the region (inset). F: The bridging vein is untethered to increase the working zone for the interhemispheric corridor. $\mathbf{G}$ and $H$ : Two sutures are placed on the superior aspect of the falx to facilitate gentle dural sinus retraction. I: The "T-shaped" incision within the falx cerebri to expose the contralateral medial hemisphere. J: Placement of additional sutures on the falcine dural flaps (inset) to increase the transfalcine working angle and corridor to the contralateral atrium. K: Corresponding intraoperative image for exposing the left precuneus. L: Following a corticotomy within the precuneus, we exposed the choroid plexus $\left(^{*}\right)$ within the contralateral atrium. Copyright The Neurosurgical Atlas (A-J), Aaron A. Cohen-Gadol, MD, MSc. Used with permission. Figure is available in color online only.

cisions within the falx are completed. Cottonoid patties protect the contralateral unaffected hemisphere while a T-shaped incision in the falx is performed as guided by neuronavigation to provide access to the precuneus over the lesion (Fig. 1I). The horizontal part of the "T-shaped" incision is made near the inferior aspect of the superior sagittal sinus, whereas the vertical portion of the incision is extended until the inferior sagittal sinus is coagulated and cut. This maneuver creates 2 dural flaps that can be retracted with retention sutures over 2 cottonoids to adequately expose the contralateral medial parietooccipital lobe (Fig. 1J and K). Resection of the lesion can then progress through a cortical incision within the precuneus to reach the periatrial region, remove the pathology at hand, and expose the choroid plexus in the atrium (Fig. 1L).

Certain details must be kept in mind to avoid complications during preparation of the contralateral interhemispheric corridor. It is important to avoid injury to the straight sinus during completion of the "T-shaped" incision within the falx, especially for the exposure of more posteriorly located lesions. If the vertical incision within the falx approaches the straight sinus as guided by neuronavigation, this vertical incision is completed in an oblique fashion from the posterior to anterior direction to protect the straight sinus along the inferior aspect of the vertical falcine incision. Using this methodology, as the falcine flaps are reflected, the more posterior regions of the precuneus are generously exposed.

After resection of the lesion, the falcine retraction sutures are removed. The falcine dural flaps are not sutured to each other to reconstruct the falx. It is important to emphasize the importance of neuronavigation for planning of the craniotomy flap location, protecting the parasagittal bridging veins, and confirming operative trajectory. Two patients underwent MR venography in addition to their preoperative MRI. In these cases, based on the latter study, we suspected numerous veins near the trajectory of our approach; the former study guided us to move our craniotomy slightly more posteriorly. Although we did not routinely use a preoperative catheter or MR venography 
to localize the bridging veins, we recommend the use of these modalities to prevent any risk to the parasagittal veins. We did not use tractography fused with neuronavigation to avoid subcortical functional white matter tracts.

Temporary postoperative external ventricular drainage was not used in any of our patients because all the blood products and debris were cleared from the ventricular system before closure. In 2 patients with slightly more posterior extension of their lesions, fluorescence angiography was used to localize the exact location of the straight sinus before the vertical portion of the incision within the falx was performed.

\section{Results}

Relevant patient information is summarized in Table 1. The mean age of patients at the time of surgery was 39 years (range 11-64 years). Six (43\%) of 14 patients were female. Pathology included 6 AVMs, 4 gliomas (2 glioblastomas, 1 anaplastic oligodendroglioma, and 1 oligoastrocytoma), 2 meningiomas (1 atypical), 1 metastatic lesion (squamous-cell lung cancer), and 1 gray matter heterotopia. The average lesion size was $25 \mathrm{~cm}^{3}$ (range $1-132 \mathrm{~cm}^{3}$ ). The most common presenting symptom was headache (10 of 14,71\%), followed by emesis and vision changes ( 3 of 14, $21 \%$ for each). Other presenting symptoms included altered mental status ( 2 of $14,14 \%$ ), aphasia ( 2 of 14, 14\%), memory loss, worsening seizures, loss of consciousness, and alexia ( 1 of 14, 7\% for each). One lesion (heterotopia) was intentionally subtotally resected; of the remaining 13 patients (93\%), 95\% or greater resection was achieved, whereas gross-total resection was accomplished in 7 (54\%) of 13 based on postoperative MRI. One patient underwent awake cortical mapping of the posterior medial frontal and anterior parietal cortices for resection of his low-grade glioma located along the medial wall of the atrium and extending along the medial frontoparietal region just inferior to the sensorimotor cortex.

There was 1 approach-related temporary complication that lasted 2 weeks: a distal left lower-extremity weakness related to the side of the approach $(1$ of $14,7 \%)$ in a patient who harbored a giant meningioma. Four patients $(29 \%)$ had postoperative complications attributable to microsurgical resection of their lesion. Two (14\%) had postoperative seizures controlled with a single medication; 3 (21\%) had new postoperative visual field deficits; and 1 patient (7\%) suffered from both a postoperative seizure and visual field defect. These visual field deficits may have been caused by aggressive coagulation of the choroid plexus within the atrium and adjacent ependymal en passage vessels due to bleeding from associated highly vascular lesions. At last follow-up (mean 4.6 months, range 0.5-19.6 months), 1 patient (7\%) had died because of complications of his underlying malignancy unrelated to his cranial surgery, 2 patients $(14 \%)$ demonstrated a GOS score of 4 , and $11(79 \%)$ manifested a GOS score of 5 . None of our patients required postoperative CSF diversion.

None of the patients in this series required fixed retractors for exposure and resection of their tumor masses. The "cross-court" working angle and dynamic retraction provided by the suction apparatus and bipolar forceps af- forded an ample working zone to handle highly vascular and neoplastic lesions. Importantly, we employed dynamic retraction by using bipolar forceps to provide additional axes of freedom, especially along the edges of the corticotomy and lateral aspects of the atrium for navigation of dissecting instruments and resection of these deep-seated lesions. In all cases, the atrium and the corresponding choroid plexus were readily exposed.

\section{Illustrative Cases \\ Case 2}

A 30-year-old woman presented with a severe sudden headache and left-sided homonymous hemianopsia (Table 1). Diagnostic tests revealed a hemorrhagic right trigonal AVM (Fig. 2A-D). Following endovascular embolization of the AVM, the patient underwent a left-sided parasagittal craniotomy and interhemispheric transfalcine approach to expose the lesion through a corticotomy in the precuneus cortex. Placement of a lumbar drain and CSF drainage allowed for generous relaxation of the unaffected left hemisphere with minimal retraction for its mobilization to access the contralateral periatrial region and resect the AVM. The interhemispheric transfalcine approach facilitated an adequate exposure of the AVM and proximal control over the choroidal and posterior cerebral artery feeders early on with minimal normal brain transgression.

We identified the main draining vein from the AVM along the medial parietooccipital region and splenium and protected it during resection. In addition, this route afforded a variety of working angles to manipulate the vascular lesion without a need for fixed retractors. The interhemispheric corridor, unlike the transcortical route, provided ample landmarks to orient us to the relevant nearby structures during microsurgery. Postoperative angiography and head CT confirmed removal of the AVM (Fig. 2E and F) as well as no injury to the contralateral hemisphere (Fig. $2 \mathrm{G})$. At 0.6 months of follow-up, Goldmann perimetry visual field evaluation demonstrated resolution of the patient's preoperative deficits.

\section{Case 1}

A 55-year-old man presented with a 6-week history of headaches and partial right-sided hemianopsia (Table 1). Preoperative imaging demonstrated a left occipital/peritrigonal mass suspected to be a high-grade glioma with extensive vasogenic edema (Fig. 3A-C). A right-sided PITTA was planned to avoid the optic radiations during removal of the tumor. Exposure of the superiorly located enhancing cyst wall would have required significant temporal lobe retraction through the lateral approach, potentially placing the cortical speech areas at risk.

Intraoperatively, this route allowed exposure of the tumor and associated cyst as it came closest to the cortical surface of the precuneus with minimal normal brain resection (Fig. 3D-H). One month after surgery, the patient's visual field deficit had improved. Gross-total resection of this histologically confirmed high-grade glioma was evident on MRI (Fig. 3I-K) immediately after surgery. 


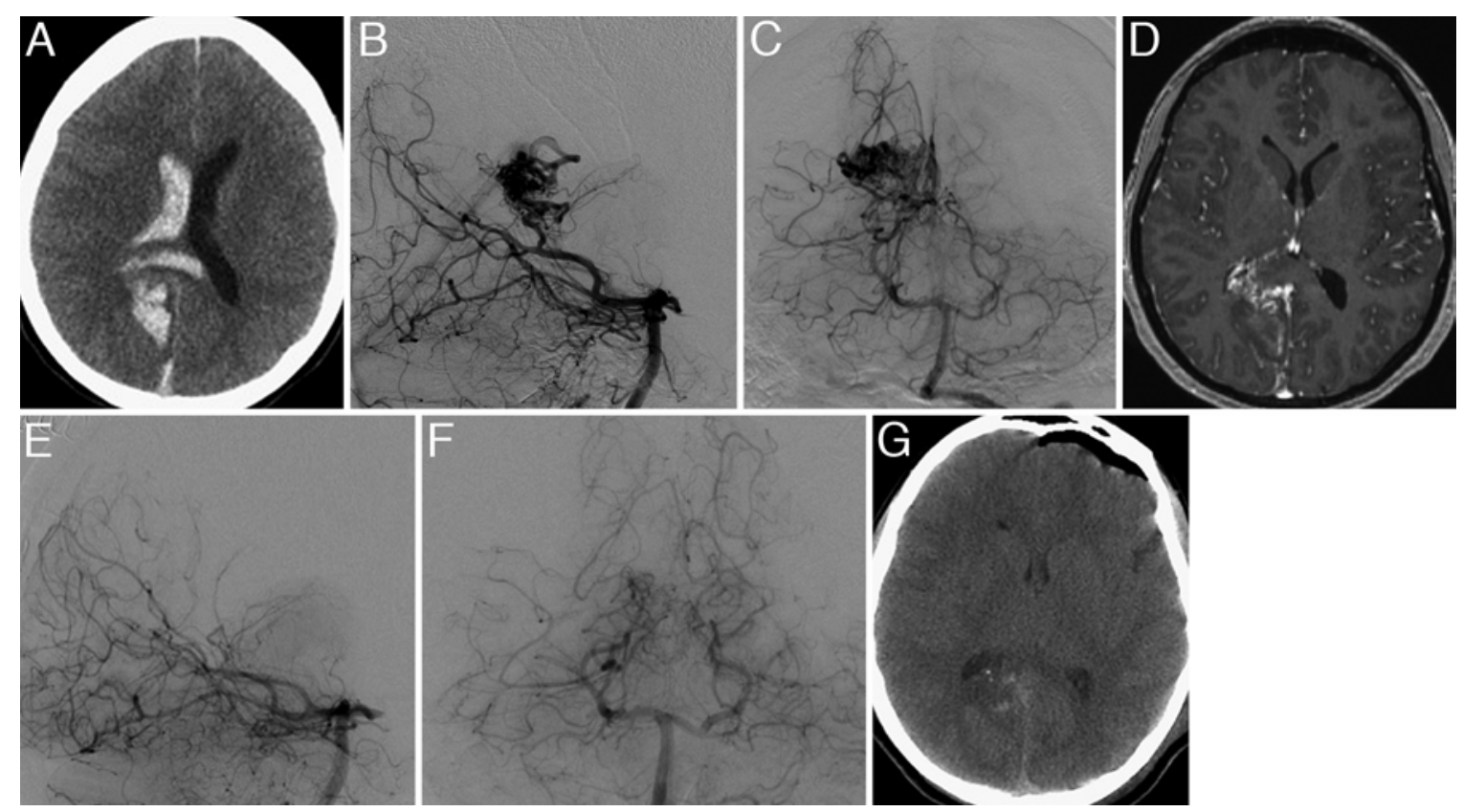

FIG. 2. Case 2. Axial CT scan of the head (A) reveals a right periatrial hemorrhagic lesion with intraventricular extension of hemorrhage. Lateral (B) and anteroposterior (C) late arterial phases of vertebral angiography demonstrate a right periatrial or trigonal AVM. An axial T1-weighted MR image with contrast (D) reveals the exact location of the periatrial AVM. We excised the AVM using the PITTA. Lateral (E) and anteroposterior (F) early arterial phases of vertebral angiography reveal gross-total removal of the AVM. An axial postoperative CT scan (G) excluded any unexpected injury to either posterior hemisphere.

\section{Discussion}

The trigone or atrium of the lateral ventricle is a triangular area defined by the junction of body, temporal, and occipital horns of the ventricle. It is a well-recognized, albeit rare $(<1 \%)$ site of AVMs and tumors such as choroid plexus papillomas and meningiomas. ${ }^{4,5} \mathrm{~A}$ variety of tumors, such as gliomas and metastases, also originate in the peritrigonal area and extend into the atrium. Lesions in this area typically present with hemorrhage, seizures, visual disturbances, and mental status change. ${ }^{1,2}$

Surgeons have studied various operative corridors to the periatrial region that allow complete microsurgical resection while minimizing risk to the surrounding structures. Kawashima et al. ${ }^{13}$ described 3 general routes: anterior transsylvian, posterior transcortical/transcallosal, and lateral trans- or subtemporal. In our opinion, the anterior transsylvian approach provides a very narrow corridor to handle highly vascular lesions and places motor fibers and optic radiations at risk. The ipsilateral posterior transcortical/transcallosal approach requires significant brain retraction to reach laterally located atrium. The transtemporal approaches place optic radiations lining the lateral wall of the trigone at risk..$^{19}$ An alternative route is the subtemporal corridor through the inferior temporal or occipitotemporal gyrus. This route is associated with less risk of speech and visual field disturbances; however, excessive temporal lobe retraction and traction on the vein of Labbe are especially problematic for the dominant hemisphere. . $^{13,25,31}$

Other surgeons have advocated the posterior middle temporal gyrus approach. ${ }^{3,11,14,15,28}$ This approach permits an early access to anterior choroidal arteries and good exposure to tumors extending into the temporal horn; howev- er, the risks include injury to the optic radiations and language deficits in the dominant hemisphere. ${ }^{21}$ Other risks of the transtemporal approach include aphasia, agraphia and alexia, and visual spatial apraxia. Current morbidity rates with the use of neuronavigation for transtemporal routes range from $0 \%$ to $20 \% .^{12}$

Other operators ${ }^{11}$ have preferred an ipsilateral parietooccipital interhemispheric approach. This approach was developed by Kempe and Blaylock. ${ }^{14}$ Visual field deficits ranging from $20 \%$ to $60 \%$ have been reported with this approach, ${ }^{22}$ and Menon et al. ${ }^{21}$ found other complications, including new motor deficits ( 3 of 15$)$, seizures ( 2 of 15 ), and dysphasia ( 1 of 15). ${ }^{21}$ Others have recommended use of the posterior transcallosal approach. ${ }^{11,15,28} \mathrm{McDermott}^{20}$ developed a modification of this route using a contralateral transcallosal trajectory. ${ }^{20} \mathrm{He}$ described transection of the inferior one to two-thirds of the falx and inferior sagittal sinus followed by no more than a $2-\mathrm{cm}$ callosotomy. This approach allows the surgeon to access lesions while minimizing transgression of the cortex and lateral white matter tracts. It also circumvents some of the shortcomings and risks of other described operative corridors. However, the challenges of traversing the corpus callosum for access to the trigone include the limitations of a narrow operative corridor and the risk of disconnection syndrome.

The ipsilateral posterior interhemispheric route is the standard and most commonly used approach for resection of medial periatrial lesions. Placement of an external ventricular drain within the occipital horn will deflate the ventricle, minimize hemispheric retraction, and allow exposure of the precuneus. Based on our experience, the PITTA provides a more "cross-court" trajectory to the lat- 

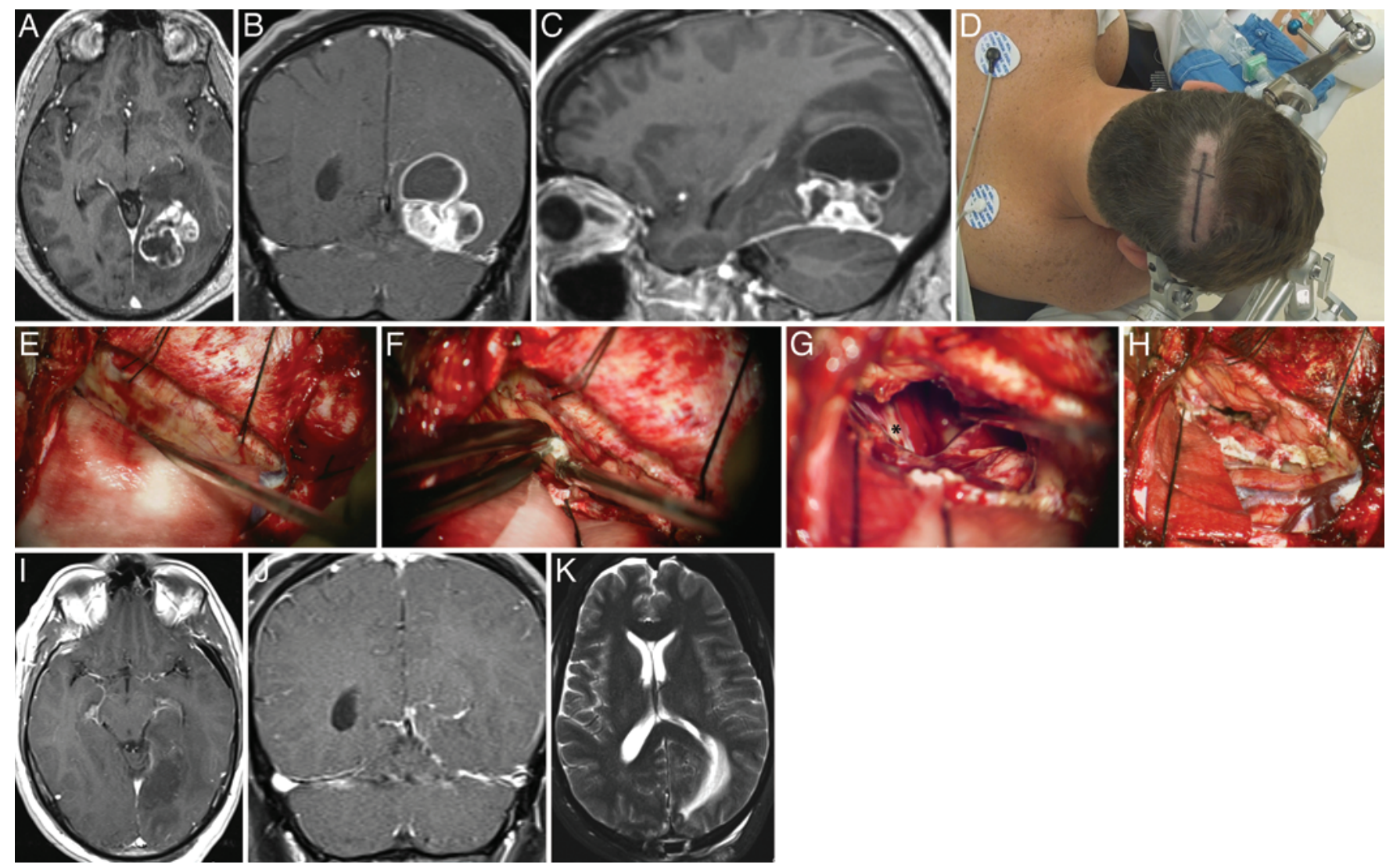

FIG. 3. Case 1. Axial (A), coronal (B), and sagittal (C) T1-weighted MR images with contrast reveal a large heterogeneously enhancing mass in the medial parietooccipital region with associated edema. The contralateral hemisphere is placed in the dependent position (D). Retention sutures are used on the falx to increase the interhemispheric working zone (E). The "T-shaped" incision in the falx has been completed and the retraction sutures on the falcine dural flaps assist with extension of the transfalcine operative angles. Corticotomy within the precuneus exposes the tumor with minimal disruption of normal cortex (F). Contralateral tentorium (asterisk) is evident at the end of tumor resection (G). A demagnified view of the operative corridor is shown in which 1 of the falcine sutures has been removed $(\mathrm{H})$. Postoperative axial $(\mathrm{I})$ and coronal $(\mathrm{J})$ T1-weighted MR images with contrast demonstrate gross-total resection of the mass. The axial T2-weighted MR image shows no evidence of retraction injury to the "approach" or right hemisphere (K). Figure is available in color online only.

eral aspect of the lesion while minimizing the degree of hemispheric retraction. We have attempted to describe this principle in Fig. 4. Please see Video 1 for further description of our technique.

VIDEO 1. Clip showing technical aspects of the PITTA. Copyright Aaron A. Cohen-Gadol. Published with permission. Click here to view with Media Player. Click here to view with Quicktime.

One modification of the posterior transcortical/transcallosal approach is the ipsilateral posterior interhemispheric transprecuneus approach for meningiomas located within the trigone of the dominant hemisphere..$^{23,32}$ The precuneus is involved in self-awareness, episodic memory, and premotor visualization/imagery. ${ }^{18}$ Injury to the precuneus is associated with temporary deficits. ${ }^{7,16,29}$ Although this approach allows the surgeon to avoid optic radiations and violation of the functional temporal cortex, it affords only a narrow working corridor and requires ipsilateral brain retraction. ${ }^{29}$ The advantages and limitations of the operative corridors to the trigone are summarized in Table 2.

One solution to this narrow operative corridor is expansion of the working angles through the "cross-court" route, i.e., the operator starts the surgical approach and interhemispheric dissection contralateral to the lesion and works across the midline, as has been described via a contralateral transcallosal approach. ${ }^{17}$ Wang et al. ${ }^{30}$ used a cadaveric model to modify the classic interhemispheric transprecuneus approach by performing a contralateral craniotomy and reaching the ipsilateral precuneus through a transfalcine method. In their specimens, a larger working angle was available (mean $44.5^{\circ}$ ) as compared with those of the classic ipsilateral approach (mean $25.8^{\circ}$ ). This method decreases the risk of visual and speech deficits associated with the temporal transcortical approach and the risks of somatosensory and visual deficits associated with the transcortical parietooccipital approach. They believed this new method improves the working angle and avoids excessive ipsilateral hemispheric retraction-both critical for minimizing adverse effects and handling vascular lesions in the region. We have applied the principles of their technique to our patients to facilitate expanding the operative corridor while minimizing brain transgression. 


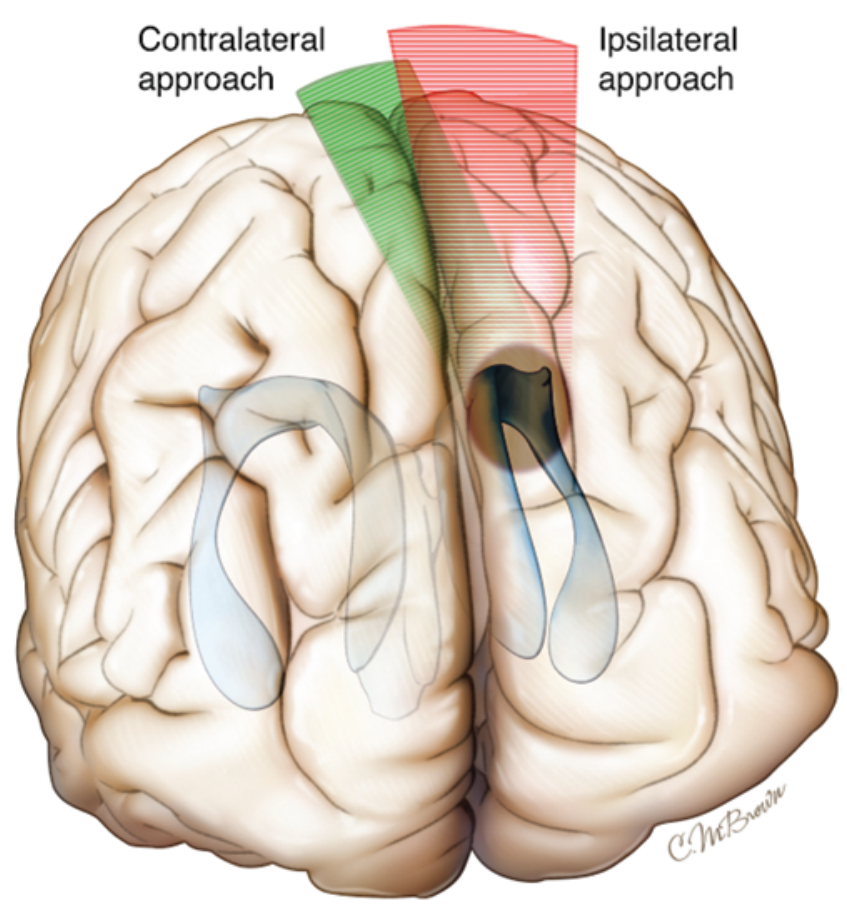

FIG. 4. Based on our experience, the PITTA (green) provides a more "cross-court" trajectory to the lateral aspect of the lesion than the ipsilateral approach (red) while minimizing the degree of hemispheric retraction. Copyright The Neurosurgical Atlas, Aaron A. Cohen-Gadol, MD, MSc. Used with permission. Figure is available in color online only.

With all of our cases in this series, patients were placed in the three-quarter prone position. The "approach" or unaffected hemisphere was placed in the dependent position, allowing gravity retraction to aid with exposure. The parasagittal bridging veins were relatively easy to untether to provide enough interhemispheric exposure. We used navigation to avoid placing the craniotomy centered over the important parasagittal veins. Retraction sutures along the superior falx facilitated superior sagittal sinus rotation and gentle mobilization to extend the interhemispheric corridor for contralateral or "cross-court" visualization. In addition, sutures on the falcine flaps further broadened exposure at the depth of the field toward the atrium.

The lesions amenable to the above-described approach are rare as they are mostly small and peri/intraventricular, often involving the medial wall of the ventricle and thus asymptomatic. In the case of peritrigonal AVMs and vascular tumors, critical arterial feeders originate from the posterior cerebral artery, the anterior choroidal artery, the posterior choroidal artery, the pericallosal and splenial arteries, and the drainage system that leads to the galenic system. ${ }^{2}$ Transtemporal or other transcortical traditional approaches may carry a high operative risk, partly because the surgeon has limited control over the feeding vessels early in the operation. ${ }^{27}$ Alternatively, PITTA provides a more direct route and early control over the arterial feeders and venous drainage systems. In addition, the PITTA allows a more generous exposure of the lateral aspect of the AVM so the operator can stay on the outside border of the lesion and manage problematic deep white matter feeders. This generous exposure is especially important in the case of AVMs. Furthermore, meningiomas in the atrium of the lateral ventricle can be devascularized early in their dissection due to a direct exposure of their feeders from the choroid plexus (Fig. 5). Based on our experience, large lesions may be accessed safely and efficaciously with this approach.

Unfortunately, 3 of our patients suffered from visual worsening due to postoperative ischemic lesions. These were the only cases during which the atrial choroid plexus (associated with 2 patients with AVMs and 1 with tumor) was aggressively coagulated due to significant bleeding from the choroid plexus that was intimately associated with these vascular lesions. Most likely, we injured the subependymal arteries by coagulating the choroid plexus. Saito and colleagues have also previously reported this complication..$^{24}$ Based on our experience, we advise against significant manipulation and coagulation of the atrial choroid plexus if possible.

TABLE 2. A summary of advantages and disadvantages of the described approaches to atrial lesions

\begin{tabular}{|c|c|c|}
\hline Approach & Advantages & Disadvantages \\
\hline Anterior distal transsylvian & $\begin{array}{l}\text { Simpler patient positioning; early control over middle cere- } \\
\text { bral artery feeders }\end{array}$ & $\begin{array}{l}\text { Risk of motor, visual, or hearing deficit; postop } \\
\text { seizures }\end{array}$ \\
\hline Transcortical parietooccipital & Avoids motor and speech areas & $\begin{array}{l}\text { Risk of parietal lobe syndrome, vision deficit; } \\
\text { postop seizures; long working distance; late } \\
\text { access to vascular pedicle }\end{array}$ \\
\hline Subtemporal/transtemporal & $\begin{array}{l}\text { Shorter working distance; early access to vascular pedicle; } \\
\text { exposure of temporal horn }\end{array}$ & $\begin{array}{l}\text { Risk of speech and vision deficits; subtemporal } \\
\text { approach carries risk of retraction and vein of } \\
\text { Labbe injury; postop seizures }\end{array}$ \\
\hline $\begin{array}{l}\text { Posterior interhemispheric } \\
\text { transcallosal }\end{array}$ & $\begin{array}{l}\text { Avoids optic radiations, temporal and parietal cortices; mini- } \\
\text { mizes postop seizures; early access to vascular pedicle }\end{array}$ & $\begin{array}{l}\text { Risk of disconnection syndromes; long working } \\
\text { distance }\end{array}$ \\
\hline $\begin{array}{l}\text { Posterior interhemispheric } \\
\text { ipsilateral transprecuneus }\end{array}$ & $\begin{array}{l}\text { Avoids optic radiations, temporal and parietal cortices; early } \\
\text { access to vascular pedicle }\end{array}$ & $\begin{array}{l}\text { Narrow working angle; significant ipsilateral hemi- } \\
\text { spheric retraction; long working distance }\end{array}$ \\
\hline PITTA & $\begin{array}{l}\text { Wider operative corridor; minimal retraction; avoids optic } \\
\text { radiations, temporal and parietal cortices; early access to } \\
\text { vascular pedicle }\end{array}$ & $\begin{array}{l}\text { Risk of injury to contralateral hemisphere; long } \\
\text { working distance }\end{array}$ \\
\hline
\end{tabular}



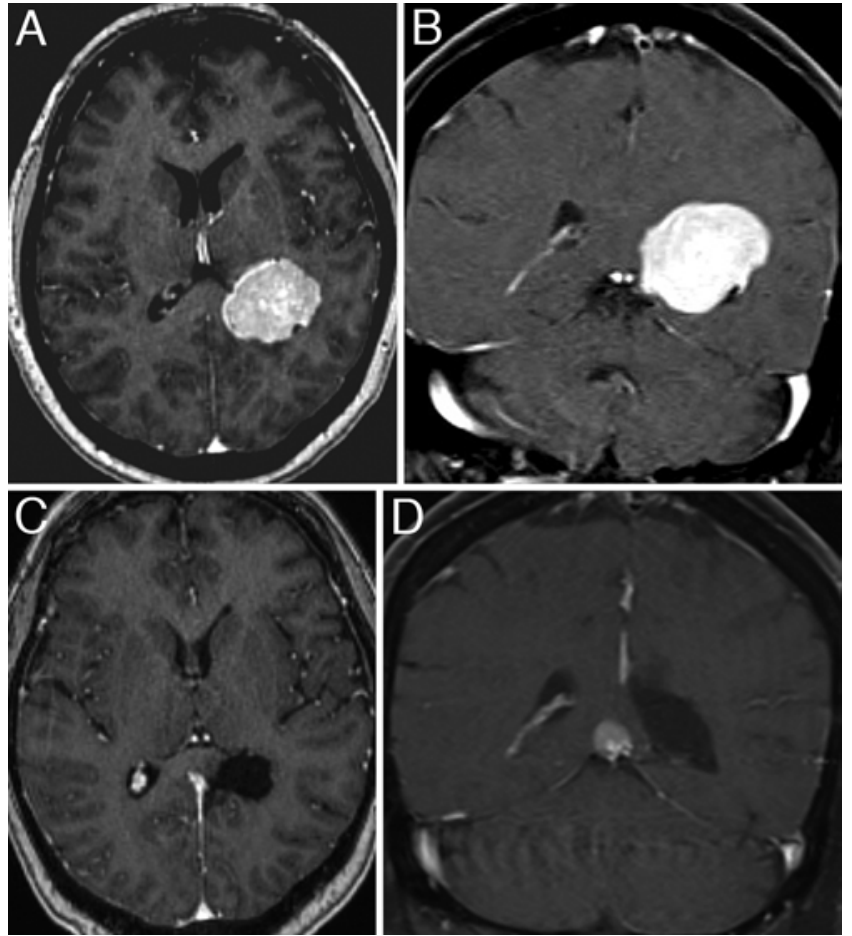

FIG. 5. Case 5. Axial (A) and coronal (B) MRI T1-weighted sequences with contrast show a left atrial meningioma that was resected using the PITTA. Early vascular control over the choroid plexus feeders to the tumor allowed safe and efficient resection of this highly vascular atypical meningioma ( $C$ and $\mathrm{D})$.

\section{Limitations of the Study}

Despite its potential advantages, there are limitations and concerns regarding our approach. The contralateral unaffected hemisphere is manipulated and draining veins are placed at risk. Venous infarction in the normal hemisphere is unacceptable as it can be catastrophic. One obvious circumstance in which to consider our approach would be in cases where there is a paucity of parasagittal bridging veins on the unaffected hemisphere as compared with the ipsilateral affected hemisphere, or the presence of edema in the ipsilateral hemisphere preventing its mobilization. In addition, the working distance in our approach is long and therefore the operation is technically challenging. Because the PITTA is also technically demanding due to its challenging oblique working angles and depth, unfamiliar anatomy, and the need for additional handling of normal hemisphere, it should be used selectively in experienced hands. More commonly employed routes such as the ipsilateral posterior interhemispheric corridor may be a reasonable alternative consideration.

This series contains heterogeneous groups of pathologies, and therefore the operative metrics described to compare various tumors and AVMs treated using this approach may be difficult to interpret. Future larger studies with inclusion of similar pathologies would be needed to investigate the advantages of the PITTA. Furthermore, only patients who demonstrated visual field deficits on detailed confrontational examination underwent ophthalmological evaluation; such a process is another limitation of our study as ophthalmological evaluation was not available in all patients.

\section{Conclusions}

Based on our experience, the posterior interhemispheric transfalcine approach to precuneus and trigonal lesions is a reasonable alternative to previously described approaches. This modification of the interhemispheric approach reduces the need for significant ipsilateral cortical resection/ retraction while improving the operator's working angles. This series demonstrates that through appropriate patient selection, this alternative route can be applied safely and effectively to a wide variety of pathologies within and medial to the trigone of the lateral ventricle.

\section{References}

1. Barrow DL, Dawson R: Surgical management of arteriovenous malformations in the region of the ventricular trigone. Neurosurgery 35:1046-1054, 1994

2. Batjer H, Samson D: Surgical approaches to trigonal arteriovenous malformations. J Neurosurg 67:511-517, 1987

3. Criscuolo GR, Symon L: Intraventricular meningioma. A review of 10 cases of the National Hospital, Queen Square (1974-1985) with reference to the literature. Acta Neurochir (Wien) 83:83-91, 1986

4. Delfini R, Acqui M, Oppido PA, Capone R, Santoro A, Ferrante L: Tumors of the lateral ventricles. Neurosurg Rev 14:127-133, 1991

5. Ellenbogen RG: Transcortical surgery for lateral ventricular tumors. Neurosurg Focus 10(6):E2, 2001

6. Ellenbogen RG, Scott RM: Transfalcine approach to a callosomarginal artery aneurysm. Neurosurgery 29:140-143, 1991

7. Fletcher PC, Frith CD, Baker SC, Shallice T, Frackowiak RS, Dolan RJ: The mind's eye-precuneus activation in memoryrelated imagery. Neuroimage 2:195-200, 1995

8. Goel A: Transfalcine approach to a contralateral hemispheric tumour. Acta Neurochir (Wien) 135:210-212, 1995

9. Heros RC: Brain resection for exposure of deep extracerebral and paraventricular lesions. Surg Neurol 34:188-195, 1990

10. Heros RC: Editorial. Contralateral approach to the atrium of the lateral ventricle. J Neurosurg 113:947-948, 2010

11. Jun CL, Nutik SL: Surgical approaches to intraventricular meningiomas of the trigone. Neurosurgery 16:416-420, 1985

12. Juretschke FR, Güresir E, Marquardt G, Berkefeld J, Rosahl $\mathrm{S}$, Klisch J, et al: Trigonal and peritrigonal lesions of the lateral ventricle-surgical considerations and outcome analysis of 20 patients. Neurosurg Rev 33:457-464, 2010

13. Kawashima M, Li X, Rhoton AL Jr, Ulm AJ, Oka H, Fujii $\mathrm{K}$ : Surgical approaches to the atrium of the lateral ventricle: microsurgical anatomy. Surg Neurol 65:436-445, 2006

14. Kempe LG, Blaylock R: Lateral-trigonal intraventricular tumors. A new operative approach. Acta Neurochir (Wien) 35:233-242, 1976

15. Konovalov ANFY, Belousawa OB: Intraventricular meningiomas, in Schmidek HH (ed): Meningiomas and Their Surgical Management. New York: WB Saunders, 1991, pp 364-374

16. Krause BJ, Schmidt D, Mottaghy FM, Taylor J, Halsband U, Herzog H, et al: Episodic retrieval activates the precuneus irrespective of the imagery content of word pair associates. A PET study. Brain 122:255-263, 1999

17. Lawton MT, Golfinos JG, Spetzler RF: The contralateral transcallosal approach: experience with 32 patients. Neurosurgery 39:729-735, 1996 
18. Lou HC, Luber B, Crupain M, Keenan JP, Nowak M, Kjaer TW, et al: Parietal cortex and representation of the mental Self. Proc Natl Acad Sci U S A 101:6827-6832, 2004

19. Mahaney KB, Abdulrauf SI: Anatomic relationship of the optic radiations to the atrium of the lateral ventricle: description of a novel entry point to the trigone. Neurosurgery 63 (4 Suppl 2):195-203, 2008

20. McDermott MW: Intraventricular meningiomas. Neurosurg Clin N Am 14:559-569, 2003

21. Menon G, Nair S, Sudhir J, Rao R, Easwer HV, Krishnakumar K: Meningiomas of the lateral ventricle - a report of 15 cases. Br J Neurosurg 23:297-303, 2009

22. Nakamura M, Roser F, Bundschuh O, Vorkapic P, Samii M: Intraventricular meningiomas: a review of 16 cases with reference to the literature. Surg Neurol 59:491-504, 2003

23. Nishizaki T, Ikeda N, Nakano S, Okamura T, Abiko S: Occipital inter-hemispheric approach for lateral ventricular trigone meningioma. Acta Neurochir (Wien) 151:1717-1721, 2009

24. Saito R, Kumabe T, Sonoda Y, Kanamori M, Mugikura S, Takahashi S, et al: Infarction of the lateral posterior choroidal artery territory after manipulation of the choroid plexus at the atrium: causal association with subependymal artery injury. J Neurosurg 119:158-163, 2013

25. Santoro A, Salvati M, Frati A, Polli FM, Delfini R, Cantore G: Surgical approaches to tumours of the lateral ventricles in the dominant hemisphere. J Neurosurg Sci 46:60-65, 2002

26. Shaw A: Fibrous tumour in the lateral ventricle of the brain, boney deposits in the arachnoid membrane of the right hemisphere. Trans Pathol Soc Lond 5:18-21, 1854

27. Spetzler RF, Martin NA: A proposed grading system for arteriovenous malformations. J Neurosurg 65:476-483, 1986

28. Tew JM, Larson, JJ: Intraventricular meningioma, in Kaye AH, Black PM (eds): Operative Neurosurgery. London: Churchill Livingstone, 2000, pp 575-585

29. Tokunaga K, Tamiya T, Date I: Transient memory disturbance after removal of an intraventricular trigonal meningioma by a parieto-occipital interhemispheric precuneus approach: Case report. Surg Neurol 65:167-169, 2006

30. Wang S, Salma A, Ammirati M: Posterior interhemispheric transfalx transprecuneus approach to the atrium of the lateral ventricle: a cadaveric study. J Neurosurg 113:949-954, 2010

31. Wang X, Cai BW, You C, He M: Microsurgical management of lateral ventricular meningiomas: a report of 51 cases. Minim Invasive Neurosurg 50:346-349, 2007

32. Yaşargil MG: Microneurosurgery. Stuttgart: Thieme, 1987

33. Zaidi HA, Chowdhry SA, Nakaji P, Abla AA, Spetzler RF: Contralateral interhemispheric approach to deep-seated cavernous malformations: surgical considerations and clinical outcomes in 31 consecutive cases. Neurosurgery 75:80-86, 2014

34. Zhu W, Xie T, Zhang X, Ma B, Wang X, Gu Y, et al: A solution to meningiomas at the trigone of the lateral ventricle using a contralateral transfalcine approach. World Neurosurg 80:167-172, 2013

\section{Author Contributions}

Conception and design: all authors. Acquisition of data: CohenGadol, Bohnstedt, Kulwin. Analysis and interpretation of data: all authors. Drafting the article: all authors. Critically revising the article: all authors. Reviewed submitted version of manuscript: all authors. Approved the final version of the manuscript on behalf of all authors: Cohen-Gadol. Study supervision: Cohen-Gadol.

\section{Supplemental Information Videos}

Video 1, Media Player. http://mfile.akamai.com/21490/wmv/ digitalwbc.download.akamai.com/21492/wm.digitalsource-naregional/jns14-847_video_1.asx.

Video 1, Quicktime. http://mfile.akamai.com/21488/mov/ digitalwbc.download.akamai.com/21492/qt.digitalsource-global/ jns14-847_video_1.mov.

\section{Correspondence}

Aaron A. Cohen-Gadol, Goodman Campbell Brain and Spine, Indiana University Department of Neurosurgery, 355 W. 16th St., Ste. 5100, Indianapolis, IN 46202. email: acohenmd@ gmail.com. 